\title{
Corrigendum to "Effectiveness of Acupuncture for Lateral Epicondylitis: A Systematic Review and Meta-Analysis of Randomized Controlled Trials"
}

\author{
Yumei Zhou $\mathbb{D}^{1},{ }^{1}$ Yuebao Guo $\mathbb{D}^{1},{ }^{1}$ Rui Zhou $\mathbb{D}^{2},{ }^{2}$ Ping Wu $\mathbb{D}^{3},{ }^{3}$ Fanrong Liang $\mathbb{D},{ }^{3}$ \\ and Zhuoxin Yang $\mathbb{D}^{1}$

\footnotetext{
${ }^{1}$ The Fourth Clinical Medical College of Guangzhou University of Chinese Medicine, Shenzhen, Guangdong 518033, China ${ }^{2}$ College of Acupuncture and Moxibustion, Guangzhou University of Chinese Medicine, Guangzhou, Guangdong 510006, China

${ }^{3}$ College of Acupuncture and Moxibustion and Tuina, Chengdu University of Traditional Chinese Medicine, Chengdu, Sichuan 610075, China
}

Correspondence should be addressed to Fanrong Liang; acuresearch@126.com and Zhuoxin Yang; 001188@gzucm.edu.cn

Received 22 January 2022; Accepted 22 January 2022; Published 17 February 2022

Copyright (C) 2022 Yumei Zhou et al. This is an open access article distributed under the Creative Commons Attribution License, which permits unrestricted use, distribution, and reproduction in any medium, provided the original work is properly cited.

In the article titled "Effectiveness of Acupuncture for Lateral Epicondylitis: A Systematic Review and Meta-Analysis of Randomized Controlled Trials" [1], some of the references in Table 2 were formatted incorrectly. Corrected Table 2 and the references are as follows:

[13] H. Hua, "Clinical observation on the treatment of external humeral epicondylitis by hysteresis acupuncture combined with acupuncture manipulation," Journal of New Chinese Medicine, vol. 50, no. 11, pp. 196-198, 2018.

[14] H. R. Yu, "Therapeutic effect of acupuncture on 147 cases of external humeral epicondylitis," Hebei Journal of Traditional Chinese Medicine, vol. 33, no. 6, pp. 890-891, 2011.
[19] L. S. Liao and W. J. Guo, "Ironing combined with acupuncture therapy for 30 cases of tennis elbow," Journal of External Therapy of TCM, vol. 26, no. 6, pp. 10-11, 2017.

[20] X. Y. Zhang, Q. Liu and M. Huang, "Effect observation of 30 cases of tennis elbow treated by fire needle," Journal of Sichuan of Traditional Chinese Medicine, vol. 33, no. 4, pp. 168-170, 2015.

[21] M. Lin, "Effect observation of 36 cases of external humerus epicondylitis treated with elbow five needle," Shandong Journal of Traditional Chinese Medicine, vol. 30, no. 9, pp. 639-640, 2011.

[22] Y. L. Wang, "Clinical observation of electroacupuncture in treatment of refractory external 


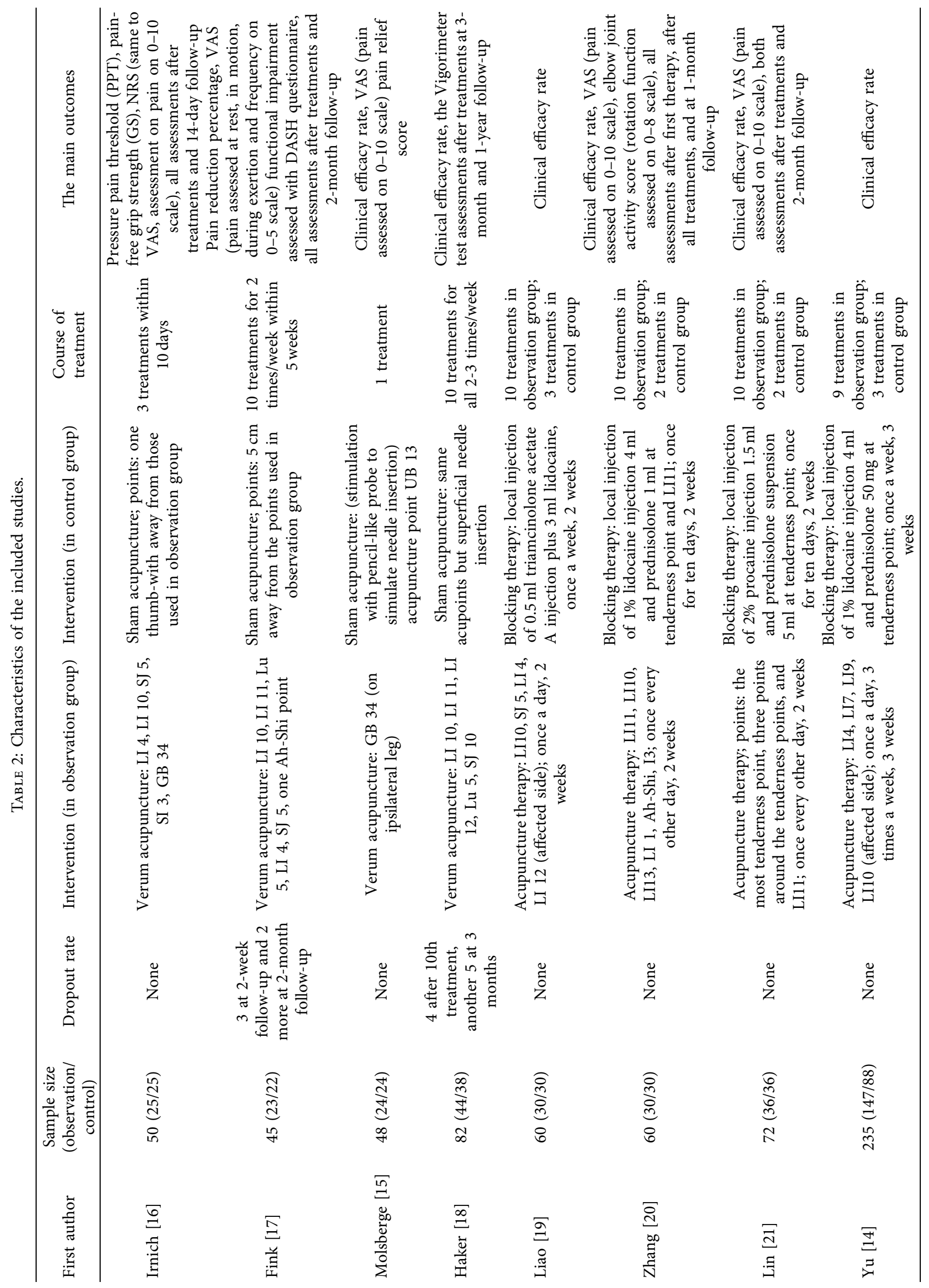




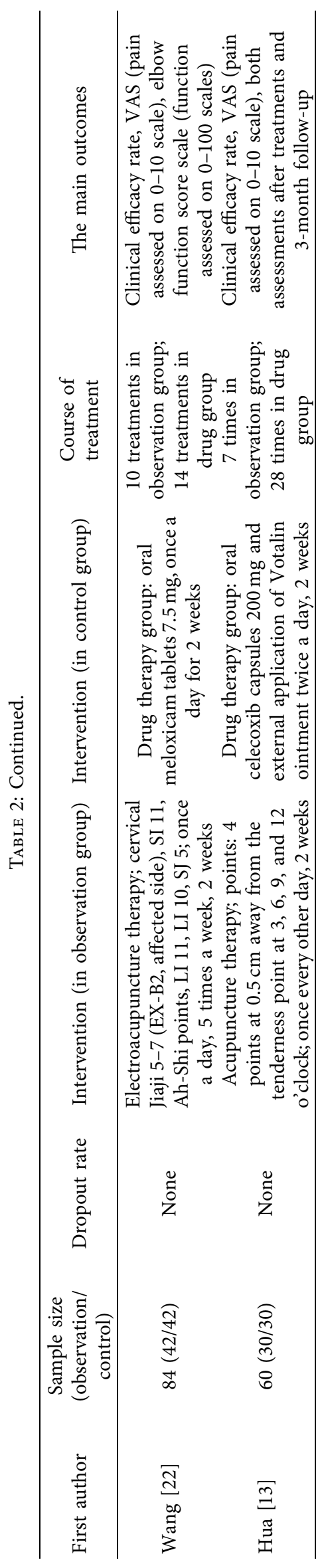


humeral epicondylitis," AsiaPacific Traditional Medicine, vol. 14, no. 8, pp. 165-166, 2018.

\section{References}

[1] Y. Zhou, Y. Guo, R. Zhou, P. Wu, F. Liang, and Z. Yang, "Effectiveness of acupuncture for lateral epicondylitis: a systematic review and meta-analysis of randomized controlled trials," Pain Research and Management, vol. 2020, Article ID 8506591, 10 pages, 2020. 